\title{
Reimagining Information Literacy teaching and learning during the COVID-19 pandemic: Research and evidence- based practice skills training redesigned for online delivery
}

\author{
Fiona Jones, Abigail Baker, Raymond A'Court and Jo Hardy \\ Macquarie University
}

\begin{abstract}
Information literacy teaching and learning for Master of Chiropractic students undertaking systematic reviews was re-envisioned from hybrid to fully online mode due to COVID-19 pandemic restrictions. Collaboration with the academic unit convener informed design and development of appropriate online learning activities and assessment for student achievement of required learning outcomes. Using flipped classroom methodology, a pre-recorded lecture demonstrated research question formulation and advanced database search strategies. Tutorial activities were redesigned for Zoom delivery using breakout rooms to replicate the collaborative aspect of face-to-face tutorials. Learning was supported by a refreshed online research guide, research consultations, and emails. Consultations were delivered via Zoom upon request for more specific advice including question formulation, search terms, choosing appropriate research databases, and advanced database search techniques. Feedback and assessments were positive and review will inform future offerings.
\end{abstract}

Keywords: case study, information literacy, systematic reviews, blended learning, collaboration

\section{Background}

The Master of Chiropractic is a two-year, full time postgraduate coursework degree offered at Macquarie University. Final year students learn how to evaluate and synthesise biomedical research via the completion of a research project. The skills learned are essential for evidence-based clinical practice. The team of four librarians who support the Faculty of Science and Engineering at Macquarie University Library was invited to teach into the course unit "Postgraduate Advanced Research 1" which has the following learning outcomes:

Critically appraise research literature on a specific clinically relevant topic and identify areas requiring future research

Effectively communicate the design of a research protocol...using verbal and written methods Demonstrate knowledge of research methods by designing a research protocol to answer a specific research question in a clinically relevant area (Macquarie University, 2020)

The unit addresses the skills needed to design and undertake a research project in the Chiropractic discipline including research question formulation, clinical practice guidelines, systematic and scoping reviews, quantitative and qualitative research design, ethics and research integrity, Cochrane's risk of bias tool (Higgins, Savovic, Page, Elbers, \& Sterne, 2019), and writing journal articles and presenting research findings. During 2019 the team of librarians designed and delivered a tailored information literacy lecture, with two complementary tutorials and a multiple-choice quiz for student assessment. Additional support was provided through an online systematic reviews research guide and post-lecture support through emails and research consultations. Consultations consisted of one-on-one, or small group, meetings with a Research Librarian to discuss specific research projects and receive individual advice and support in finding, evaluating, and using research literature. For delivery in 2020 a similar blend of face-to-face and online support was planned. In addition, the Library's systematic reviews guide (Macquarie University Library, 2020) had been substantially refreshed in a separate project and was ready to be used as a supporting online resource.

Due to the COVID-19 pandemic, staff and students were suddenly faced with significant changes to modes of teaching and learning, forms of assessment and session timetable. Chiropractic students also still needed to deliver clinical patient care to meet the requirements for successful course completion. For the library team this meant undertaking a rapid pivot to convert a successful hybrid approach to one that was fully online, while maintaining the levels of engagement and effectiveness. 


\section{Approach}

To ensure that best practice was followed in reimagining the content for an online space, the librarians conducted an environmental scan and a review of prior online offerings created by the team and other library colleagues. Options were then considered in collaboration with the academic unit convenor. These discussions and a brief review of the relevant literature highlighted two important considerations for best practice in online instruction. First was the benefits of applying a flipped classroom model. Using a flipped method "traditional lecture content is delivered through student self-instruction before class...Class time is then devoted to engaging in learning exercises, intensive practice opportunities, and learning assessment.” (Loo et al., 2016, p. 273).

Many educational institutions have embraced the flipped classroom, and those who have employed it have reported the following benefits: increased student engagement and interaction, the ability to be more hands-on and customise relevant activities, and a more welcoming environment for students to ask questions (Loo et al., 2016; Rivera, 2015). Second, the use of small groups or "breakout rooms" for discussion was recommended. In this context, breakout rooms refer to a subset of participants from the larger group being assigned to a smaller group for audio, video or text chat. They are available as a function of some web-based platforms including Zoom (Zoom Video Communications Inc, 2020) to enable more participatory learning opportunities. Benefits of small groups for online tutorials include greater engagement and interactivity with students, the opportunity for peer-support and peer-learning, and the capacity to make it easier for students to talk and feel more confident than when participating in discussions in a large group (Chandler, 2016). It was decided that both methods would be employed in the reimagining of tutorial delivery to enhance student experience.

In 2019 the lecture was delivered by two librarians face-to-face as a one-hour presentation. The content included an example of converting research questions into population, interventions, comparators, outcomes and study designs (PICOs) (Centre for Reviews and Dissemination, 2009), and then into keywords and Medical Subject Headings (MeSH) in a live demonstration of a MEDLINE® (National Library of Medicine, 2020) search. Understanding this content is essential for students to fully participate in the following tutorials. When considering how to deliver this appropriately in the changed environment, librarians considered the large amount of complex information to be communicated and concluded that a video recording would be the best approach in 2020. This flipped classroom approach enabled students to watch the lecture in their own time and re-watch to consolidate their understanding of difficult concepts prior to engaging in the tutorials. Simplicity and the limitations of technology dictated that this lecture would be recorded by one librarian using the Echo360 (Echo360, 2020) screen capture software. A media file was created using presentation slides accompanied by a voiceover. The slides included tables and annotated screenshots to illuminate search planning and strategy, and to form a resource for student referral. The chosen format meant that significant reworking of the content was not required, and the experience would not differ substantially for 2020. This lecture recording was made available to students via their online unit within the university's learning management system (LMS) (Moodle Pty Ltd, 2020). The mode of delivery also increased opportunity of access compared to 2019 when students had to attend the early morning lecture immediately prior to the tutorials then cross campus to the Library building.

The mode of delivery for the tutorials required greater consideration. The goal of the tutorials was for students to engage in activities to ground new knowledge and practice advanced search skills. They would begin with the evaluation of example search strategies and then apply this knowledge directly to their own research project by completing a worksheet to demonstrate their level of understanding. The worksheet was presented in three stages to replicate the lecture demonstration. Step one was to translate the question into PICOs format; step two required converting these into MeSH and keyword terms; and step three involved building them into a search strategy in MEDLINE®. In 2019 students split into small groups for these activities and were assisted by four librarians who directed the tasks and moved around the room to answer questions and provide guidance. In 2020 the video conferencing software Zoom was chosen to host the tutorial. The breakout rooms feature was employed to replicate the group work experience of the 2019 tutorials. In 2020 there were ninety students enrolled in the unit and expected to attend across the two scheduled tutorials. The convenor's preference was for no more than four or five students per breakout room so it was expected that at least nine breakout rooms in each tutorial may be required. For optimal outcomes it was decided that a librarian, or the convenor, would be assigned to each breakout room to help by facilitating discussion and supporting students, and staff could be moved from group to group to ensure that all students received assistance. This method required more librarians to be available than in 2019 , and another librarian was asked to assist. This brought the teaching support to five librarians and the convenor for each tutorial. On the day twenty-five students attended each tutorial which allowed for each staff member to stay in one breakout room for the full activity time. This became an advantage as the librarians were able to focus on a specific group, generating discussion, and becoming a part of their unique learning journey through the material. Staff also reported the themes and questions that arose when the 
larger group reconvened at the end of each activity. The experience would have been different if all the expected students had attended, as staff would have had to switch between groups and spend a shorter time in discussion, relying on students to stay on task. This is something to consider for future iterations, as it could have a significant effect on student learning. A possible solution would be to provide more support for groups to complete the activity independently by setting specific questions to answer and report back on.

To further support students after the tutorials they were invited to contact the librarians for individual advice if required. In 2019 this support was either by email, telephone, or research consultation. For 2020 no telephone support was available. Research consultations were provided to six individuals and two groups. Each client or group met with librarians more than once in real time consultations via Zoom. In addition, students and librarians exchanged emails to address follow-up questions. This represented significantly more engagement by the students than in 2019, when only three consultations were delivered during the same timeframe. Support included more detailed advice regarding question formulation, research database choice, selection of search terms, and the construction of database searches. A large percentage of staff time, both before and during consultations, was devoted to developing and demonstrating example MEDLINE® search strategies step-by- step. Out of scope for this paper is a discussion of the earlier project resulting in the refreshed systematic

reviews guide (Macquarie University Library, 2020). Because the refreshed guide used the steps for performing a systematic review as a scaffold for its content, it was easy to navigate for inexperienced researchers and provided another source of online support.

In 2019 a multiple-choice quiz was embedded in the LMS for online delivery. In 2020 an additional ten questions were designed to bring the bank of questions to a total of twenty. A selection of questions from this bank were included in the online assessment quiz. Questions tested the following learning outcomes:

- Students can use the acronym PICOs to identify the key concepts in a research question.

- Students can identify the steps required to perform a systematic review.

- Students can plan a comprehensive search strategy.

- Students can correctly use the "AND", "OR" Boolean Operators to construct search strategies.

- Students can use appropriate subject headings, including MeSH in search strategies.

- Students can perform a complex search in MEDLINE® on the OVID (Wolters Kluwer, 2020) platform.

\section{Feedback, Review and Reflection}

To evaluate the success of the activities and resources, the library team collected feedback from students, reviewed learning via an assessment task, and reflected on teaching experiences. The 2020 students were asked to complete a quick survey comprised of two questions. Questions were based on the "minute paper" proposed by Angelo (1993) which asks students to identify the most significant thing that they learned, as well as anything that they did not understand or was not addressed sufficiently. This method is widely used in an adapted format to collect learner feedback at Carpentries (The Carpentries, 2020a) workshops, where learners are asked to write one positive and one constructive negative comment after instruction at breaks in the program (The Carpentries, 2020b). The post-tutorial survey asked the following two questions:

1. Tell us one useful thing you learned in the Library lecture and tutorial

2. Tell us one thing that you are still unsure about or suggest an improvement

The survey was made available to students via the LMS by the convenor. Eleven responses were received. Positive responses included the following:

- "I loved watching you go through the steps live, it was very easy to follow and understand."

- "It was super interesting to learn how to effectively and efficiently search the databases, and how to save previous searches in an account"

- "The staff showed us the basics of the Boolean operators, database filters and so forth."

- "I really liked the screen shots that went step by step, super helpful to reference back to!"

- "I really liked having the guidance of the librarians in the breakout rooms"

These positive responses confirmed that the live database search demonstrations, the explanation of advanced search techniques and the support provided by online resources and librarians were all appreciated by the students. The constructive suggestions for improvement fell into the following themes: 
- More examples and support needed for choosing search terms, especially MeSH.

- Demonstration of searching on other databases.

- More search activities, including the opportunity to test understanding via exercises.

- Desire to have a similar Library session delivered earlier in the degree.

Most of these suggestions can be addressed in future offerings by extending the flipped approach. To address this, additional digital resources on search planning and database search demonstrations could be added to the LMS and the tutorials could be adjusted to focus on search planning and construction exercises. The least successful activity in the tutorials was the evaluation of example search strategies, which students found very difficult before they had constructed any searches for themselves. As an alternative, this activity could be reimagined as a short concluding quiz which may assist in the consolidation of students' newly acquired information literacy skills. Notably none of the feedback or suggestions for improvement related to the platform chosen, nor were there any issues or comments about accessing the lecture online or participating in an online tutorial. This may suggest that the students did not perceive any disadvantage with the move to the fully online environment; however, a limitation of application of the feedback received was the small sample size.

Analysis of 2020 multiple choice assessment results revealed that students had difficulty understanding the relationship between the PICOs acronym, identification of key concepts in a question, and the subsequent construction of an appropriate search strategy. They also struggled to understand the need to balance sensitivity and precision when conducting systematic literature searches and the effect that the Boolean operators AND and OR have on reducing and increasing the number of search results returned. These difficulties were similar to those observed in 2019 and indicate the need to incorporate some formative assessment to support students to consolidate their understanding. By including a short interactive quiz at the conclusion of future tutorials, students would have an opportunity to practice the skills taught and receive feedback on their performance. It is anticipated that students would then perform more favourably on the assessment quiz.

\begin{abstract}
A further measure of student capabilities and understanding may be ascertained through the sample of follow-up consultations. Compared to 2019, several students appeared to have a better grasp on the concept of Boolean searching and how to begin to construct a search strategy. However, similarly to 2019 consultations, many students had difficulty with the scope of their search strategy planning, often either relying on a small number of familiar terms or using too many broad terms that obscured their question. Another key point of instruction in both followup consultations and emails was the managing of expectations around search results. Several students expressed frustration due to retrieving a large number of results which included irrelevant papers. To remedy this, students were referred back to the systematic review principles, with the need to balance the tension between sensitivity and precision. Increased engagement via consultations and emails during 2020 enabled the librarians to address these difficulties with students as they related to their particular projects.
\end{abstract}

Reflecting on the tutorial delivery, the library team agreed that managing small group discussion online was easier with full attention being given to one small group without distractions from others in a physical room. However, it was also agreed that the Zoom breakout room format allowed for, and possibly encouraged, less engagement from the students in general. In each breakout room it was observed that at least one or more students in a group muted their audio and/or video. It cannot be determined whether this was due to technical constraints, anxiety about being seen and heard, or disengagement with the material. Other factors may be having children, pets, or other household members in the background. These reasons could be directly related to the rapid shift required with COVID-19 restrictions, but also reflect more general issues faced by students learning online in many different contexts. In any case, students who shared video and/or audio had to carry the discussion for their peers. Anecdotal feedback suggests that students may be intimidated by the experience of online discussion, in which interpreting tone and finding an opportunity to contribute are more difficult and speaking to the group feels more performative than is usual in person. It was agreed that in future, breakout rooms should begin with an introduction from each participant. This would allow staff to establish a connection with students, become aware of who is engaged and able to participate, and would provide an opportunity for students to share their specific research projects. Using these projects as examples to demonstrate information literacy concepts and techniques should encourage engagement in the discussion.

In terms of attendance, in 2019 sixty students were enrolled and only thirty-five attended the library lecture, with thirty in each of the tutorials. Poor lecture attendance impacted the tutorial activities as lecture content needed to be reviewed. In 2020 lecture content was also reviewed in the online tutorials, although feedback indicates that students would prefer to use tutorial time for activities. At the time of writing the video lecture had been viewed ninety-six times, over a cohort of ninety enrolled students. It was not possible to differentiate between pre- and 
post-tutorial viewings, however the number of views is encouraging and indicates that some students are using it as a referral resource. In 2020 twenty-five students attended each tutorial.

One highly important benefit was highlighted by all these experiences. Working alongside the convenor to embed information literacy content into the structure of a unit or course is essential to ensure a successful learning and teaching experience for everyone involved. The convenor input ensures that outcomes are aligned, content is appropriately tailored, and support is offered in a consistent and equitable manner. The quick pivot required to move this unit online opened up space to enhance the collaboration between librarian and convenor. Working with students on assessment tasks makes the activities much more relevant and increases motivation, whilst the presence of the convenor in the information literacy tutorials demonstrates their value to students.

\section{Conclusion}

The project to convert Library training and support services for Master of Chiropractic students from face-to- face to online was a success. Despite the quick turnaround time, the library team were able to recreate a suite of tailored information literacy resources and services which continued quality service provision in unprecedented circumstances. This unique situation of teaching during COVID-19 restrictions was an opportunity to be agile and flexible and consider how to create student focused learning experiences in the online environment. It was also an excellent example of the benefits of collaboration between library and academic staff in embedding information literacy content into a unit of study. The experience and learnings gained can now feed into improvements for future student cohorts. Library staff capabilities were also developed through the adaptation to new technology, and the creative approach needed to adjust to new learning environments. The team are now confident in their capabilities to provide relevant and engaging digital learning objects and training. The successful delivery of the online lecture and tutorials, the reusable digital learning objects created, and the positive student and convenor feedback, provide evidence for the viability of this approach.

\section{References}

Angelo, T. A. (1993). Classroom assessment techniques: A handbook for college teachers (2nd ed.). San Francisco: Jossey-Bass Publishers.

Centre for Reviews and Dissemination. (2009). Systematic reviews: CRD's guidance for undertaking reviews in health care. Retrieved from https://www.york.ac.uk/media/crd/Systematic Reviews.pdf.

Chandler, K. (2016). Using breakout rooms in synchronous online tutorials. Journal of Perspectives in Applied Academic Practice, 4(3), 16-23. doi:10.14297/jpaap.v4i3.216.

Echo360. (2020). Echo360. Retrieved from https://echo360.com/.

Higgins, J. P. T., Savovic, J., Page, M. J., Elbers, R. G., \& Sterne, J. A. C. (2019). Assessing risk of bias in a randomized trial. In J. P. T. Higgins, J. Thomas, J. Chandler, M. Cumpston, T. Li, M. J. Page, \& V. A. Welch (Eds.), Cochrane Handbook for Systematic Reviews of Interventions (6.0 ed.). Retrieved from http://www.training.cochrane.org/handbook.

Loo, J. L., Eifler, D., Smith, E., Pendse, L., He, J., Sholinbeck, M., . . . Dupuis, E. A. (2016). Flipped instruction for information literacy: Five instructional cases of academic librarians. The Journal of Academic Librarianship, 42(3), 273-280. doi:10.1016/j.acalib.2016.03.001.

Macquarie University. (2020). CHIR8201 Postgraduate Advanced Research 1 Unit Guide for 2020 Session 1. Retrieved from https://unitguides.mq.edu.au/unit_offerings/122917/unit_guide?full_code=CHIR8201_FHFYR_2020.

Macquarie University Library. (2020). Systematic Reviews. Retrieved from https://libguides.mq.edu.au/systematic reviews.

Moodle Pty Ltd (Producer). (2020). Moodle. Retrieved from https://moodle.org/ National Library of Medicine (Producer). (2020). Medline. [Database] Retrieved from https://www.nlm.nih.gov/bsd/medline.html.

Rivera, E. (2015). Using the flipped classroom model in your library instruction course. The Reference Librarian, 56(1), 34-41. doi:10.1080/02763877.2015.977671.

The Carpentries. (2020a). The Carpentries. Retrieved from https://carpentries.org/.

The Carpentries. (2020b). Instructor training: Building skill with feedback. Retrieved from https://carpentries.github.io/instructor-training/06-feedback/index.html.

Wolters Kluwer (Producer). (2020). Ovid. Retrieved from https://www.ovid.com/platforms/point-ofreference/ovid.html.

Zoom Video Communications Inc (Producer). (2020). Zoom Meetings. Retrieved from https://zoom.us/meetings. 
Jones, F., Baker, A., A'Court, R. \& Hardy, J. (2020). Reimagining Information Literacy teaching and learning during the COVID-19 pandemic: Research and evidence-based practice skills training redesigned for online delivery. In S. Gregory, S. Warburton, \& M. Parkes (Eds.), ASCILITE's First Virtual Conference. Proceedings ASCILITE 2020 in Armidale (pp. 112-117). https://doi.org/10.14742/ascilite2020.0132

Note: All published papers are refereed, having undergone a double-blind peer-review process.

The author(s) assign a Creative Commons by attribution licence enabling others to distribute, remix, tweak, and build upon their work, even commercially, as long as credit is given to the author(s) for the original creation.

() Jones, F., Baker, A., A’Court, R. \& Hardy, J. 2020 